\title{
La modelación matemática en la formación del ingeniero
}

\author{
Patricia Camarena Gallardo
}

\section{Resumen}

La presente conferencia incide en la modelación matemática como elemento clave en la formación del ingeniero, donde la matemática no es una meta por sí misma. Además, en muchas universidades la modelación matemática no ha sido incorporada de manera formal en los ambientes de aprendizaje, por lo que este documento presenta una estrategia didáctica para incorporar de forma consiente y formal la modelación matemática en los cursos de matemáticas, contando con una clasificación y caracterización de los mismos.

Palabras clave: Modelación, Matemáticas, Matemáticas en Contexto, Modelo, Ingeniería.

\begin{abstract}
The conference is about mathematical modeling as a goal element in the engineer preparing, where mathematics are not a goal themselves. Besides, the mathematical modeling has no included formally in the courses of many universities; this document presents a didactic proposition which included the mathematical modeling in mathematics courses in a formal and conscious way, it has a classification and characterization of mathematical models in the engineering careers.
\end{abstract}

Keywords: Modeling, Mathematics, Mathematics in Context, Model, Engineering. 


\section{Introducción}

La matemática es una asignatura con un alto índice de reprobación, elemento que es un síntoma de la problemática que la matemática representa para los estudiantes. Es un echo el poco interés que tienen los alumnos por esta rama de las ciencias, ya que no ven de manera inmediata su aplicación, ni el objeto de tener que cursarla; en buena medida, un elemento que afecta, es la desvinculación que existe entre los cursos de matemáticas y las demás asignaturas de la carrera en donde se imparten estos cursos (Camarena, 1984; 1987).

Por otro lado, cuando en su actividad profesional y laboral los egresados tienen que resolver un problema de la industria, la modelación matemática es necesaria, y los estudiantes tiene dificultades para modelar el problema ya que no han sido preparados para ello durante sus estudios universitarios.

La modelación matemática es uno de los temas que aparece en el currículo oculto de los programas académicos de estudios universitarios, ya que se supone que el egresado debe saber modelar matemáticamente $y$, en muchos planes y programas de estudio para nada se hace alusión al término "modelación matemática". En otros currículos, dentro de los objetivos de los programas de estudio, se dice que el alumno deberá saber modelar problemas de otras áreas del conocimiento, y en muy pocos currículos viene este término incluido en el temario de las asignaturas. Pero, en ningún caso se dice cómo incorporar la modelación matemática a los cursos de matemáticas, ni cómo lograr que los estudiantes modelen situaciones de otras áreas o problemas de la vida cotidiana (Camarena, 2001). e hecho, en la mayoría de los casos no existe ninguna asignatura de la ingeniería que se aboque a elaborar modelos matemáticos.

Además, resulta que los profesores de matemáticas sienten que este punto compete a los profesores de los cursos propios de la ingeniería, mientras que estos últimos presuponen que los maestros de matemáticas son quienes deben enseñar al estudiante a modelar fenómenos o problemas de la ingeniería (Camarena 1990).

Cabe mencionar que este punto de conflicto, el cómo modelar matemáticamente problemas de la vida laboral y profesional, desde la teoría de la Matemática en el Contexto de las Ciencias se considera que debe ser atendido de forma interdisciplinaria.

\section{Objetivo de la investigación}

Con la problemática descrita se plantea el objetivo de la investigación que se reporta en esta presentación. El objetivo persigue tener una estrategia didáctica que incorpore de forma consiente la modelación matemática en los cursos de matemáticas.

Como existen muchas clases de modelos matemáticos, con diversos grados de dificultad, se hace necesaria una clasificación y caracterización de los modelos matemáticos en ingeniería. 


\section{Marco teórico}

La Matemática en el Contexto de las Ciencias es la teoría en la que se fundamente la investigación. Esta teoría educativa reflexiona acerca de la vinculación que debe existir entre la matemática y las ciencias que la requieren, la matemática y las competencias laborales y profesionales, así como entre la matemática y las actividades de la vida cotidianas, ya que se persigue una matemática para la vida.

La Matemática en el Contexto de las Ciencias se fundamenta en tres paradigmas (Camarena, 1984; 1987; 1990; 1995; 2000: 2001; 2005; 2008):

- La matemática es una herramienta de apoyo y materia formativa.

- La matemática tiene una función específica en el nivel superior.

- Los conocimientos nacen integrados.

El supuesto filosófico educativo de esta teoría es que el estudiante esté capacitado para hacer la transferencia del conocimiento de la matemática a las áreas que la requieren y con ello las competencias profesionales y laborales se vean favorecidas.

La teoría se constituye por cinco fases:

- La Curricular, desarrollada desde 1984.

- La Didáctica, iniciada desde 1987.

- La Epistemológica, abordada en 1988.

- La de Formación Docente, definida en 1990.

- La Cognitiva, estudiada desde 1992.

\section{La estrategia didáctica: Matemática en Contexto}

La presente investigación incide en la fase didáctica de la teoría de la Matemática en el contexto de las Ciencias, cuya propuesta didáctica se denomina la Matemática en contexto, a través de la cual se trabaja con eventos contextualizados. Esto es, problemas y proyectos en el contexto de las demás áreas del conocimiento de los estudios del alumno, en actividades de la futura vida profesional y laboral y, en problemas de la vida cotidiana.

La Matemática en Contexto contempla nueve etapas (Camarena, 1987):

1. Analizar los textos de las demás asignaturas que cursa el estudiante.

2. Plantear el evento de las disciplinas del contexto.

3. Determinar de las variables y de las constantes del evento. 
4. Incluir los temas y conceptos matemáticos necesarios para el desarrollo del modelo

matemático y su solución.

5. Determinar el modelo matemático.

6. Dar la solución matemática del evento.

7. Determinar la solución requerida por el evento en el ámbito de las disciplinas del

contexto.

8. Interpretar la solución en términos del evento y áreas de las disciplinas del contexto.

9. Presentar en el salón de clases una matemática descontextualizada para que el estudiante sepa que es aplicable a otros campos del conocimiento y desarrolle lashabilidades que proporciona la matemática formal.

Se puede observar que la construcción del modelo matemático es un elemento central. Es central en el sentido de que sin este elemento la matemática en contexto no se logra, ni la solución del evento, es decir, para resolver el evento contextualizado es necesario construir el modelo matemático que describe el evento. Esto lleva a realizar investigaciones en la línea de modelación matemática.

En general el hablar de la Matemática en Contexto es desarrollar los cursos de matemáticas a las necesidades y ritmos que dictan los cursos de ingeniería. De hecho, la Matemática en Contexto fortalece la reorganización cognitiva de conceptos y procesos matemáticos (Camarena,1999).

A través de la Matemática en Contexto se cambia el paradigma educativo de enseñanza tradicional, ahora se trata de una enseñanza con conocimientos integrados, dando los temas de as vinculados con las demás asignaturas que cursa el alumno y presentándolas al ritmo y tiempos que son requeridos por ellos. Con la Matemática en Contexto se construyen conocimientos integrados no fraccionados, aprendizajes significativos (en el sentido que lo define Ausubel, 1970), así como conocimientos duraderos no volátiles (Camarena, 2000; 1999).

Para llevar a cabo en el ambiente de aprendizaje la estrategia didáctica de la Matemática en Contexto, es necesario que se conformen equipos de tres alumnos, en donde se identifica al líder académico, al líder emocional y al líder operativo.

También es necesario tomar en cuenta los elementos de la resolución de problemas, como son las heurísticas de Polya (1976), así como, la metacognición y las creencias que describe Santos (2000). 


\section{Los modelos matemáticos en la ingeniería}

Por la relevancia de los modelos matemáticos en la fase didáctica de la matemática en el contexto de las ciencias, a continuación se muestran los resultados de una investigación en donde se caracteriza y clasifica a los modelos matemáticos en el área de la ingeniería.

Para iniciar, se tiene que la matemática en ingeniería es un lenguaje, ya que casi todo lo que se dice en la ingeniería se puede representar a través de simbología matemática (Camarena, 1990).

Es más, el que se represente a través de la terminología matemática y se haga uso de la matemática en la ingeniería, le ayuda a la ingeniería a tener carácter de ciencia por un lado y por el otro, le facilita su comunicación con la comunidad científica de ingenieros.

Después de analizar problemas de la ingeniería reportados en los libros de texto de los estudiantes, así como problemas reales de la industria y problemas de proyectos de investigación en ingeniería, se identificó que dentro del conocimiento de la ingeniería, se tienen tres tipos de elementos que se describen matemáticamente (Camarena, 1987; 1993; 2007):

- Problemas de la ingeniería

- Objetos de la ingeniería

- Situaciones específicas que determina la ingeniería

\section{a) Problemas.}

Se quiere conocer el fenómeno de carga de un condensador (capacitor), cuya capacitancia es $\mathrm{C}$, el cual está conectado en serie con un resistor de resistencia $\mathrm{R}$, a las terminales de una batería que suministra una tensión constante $V$, este planteamiento se puede representar a través de la ecuación diferencial lineal siguiente:

$$
R \frac{d}{d t} q(t)+\frac{1}{C} q(t)=V
$$

Es de mencionar que bajo el término problema se están incluyendo los fenómenos que se presentan en la ingeniería, como la carga de un condensador, la caída libre de un cuerpo, el movimiento de un péndulo, etc. 


\section{b) Objetos.}

Considérese una señal eléctrica del tipo alterno sinusoidal, la señal es el objeto de la ingeniería el cual se representa a través de la función:

$$
f(t)=A \operatorname{sen}(t+\propto)
$$

\section{c) Situaciones.}

El condensador de carga $\mathrm{q}=\mathrm{q}(\mathrm{t})$, está totalmente descargado al inicio del problema. Esta situación se puede representar matemáticamente, tomando en cuenta que al inicio del problema $t=0$ y que la carga es una función del tiempo, como: $q(0)=0$

\section{Caracterización de los modelos matemáticos en ingeniería}

De los tres casos mencionados, los que caracterizan a los modelos matemáticos, como los concibió la investigación, son los objetos y los problemas, así la definición es: Un modelo matemático es aquella relación matemática que describe objetos o problemas de la ingeniería.

\section{Clasificación 1. Modelos de tipo estático y dinámico}

Cuando los modelos matemáticos describen objetos de la ingeniería, éstos dan origen a modelos de tipo dinámico o estático.

Los modelos dinámicos son relaciones matemáticas que constantemente, por las necesidades de la ingeniería, requieren de modificaciones matemáticas.

Los modelos estáticos son relaciones matemáticas que describen a un objeto de la ingeniería como si fuera un "apodo", es decir, matemáticamente no se hace nada más.

Como se puede observar de esta clasificación, en modelos estáticos y dinámicos, ésta está en función del uso que se le da en la ingeniería, por lo que es obvio que un modelo dado podrá ser dinámico en alguna especialidad de la ingeniería, mientras que en otra podrá ser estático.

\section{Clasificación 2. Modelos por generaciones}

Cuando los modelos matemáticos describen problemas de la ingeniería, éstos se pueden clasificar en modelos de primera generación, los cuales se obtienen de datos experimentales de la iería, como por ejemplo determinar la ley de Ohm. También se incluyen en este tipo de modelos los fenómenos de la ingeniería como la carga de un condensador, la caída libre de un cuerpo, el movimiento de un péndulo, etc. 
Si se hace uso de estos modelos de primera generación para construir nuevas relaciones, a estás se les denomina modelos de segunda generación. Como el ejemplo de una ecuación que representa el fenómeno de carga de un condensador.

A su vez, cuando se requiere modelar un problema que requiere de modelos de primera y segunda generación, éstos determinan modelos de tercera generación. Como el caso de una red eléctrica en donde se puede modelar a través de un sistema de ecuaciones diferenciales.

Cuando se hace uso de modelos de primera, segunda y tercera generación se da por origen a modelos de cuarta generación. Frecuentemente para modelar este tipo de problemas se recurre a los programas de software que realizan simulaciones, por la complejidad del problema.

Cabe hacer mención que los problemas de la ingeniería que se analizaron y que fueron detectados de los libros de texto de los estudiantes, se correlacionaron con el tipo de generación del modelo matemático.

De esta forma, los modelos matemáticos de primera y algunos de segunda generación corresponden a las ciencias básicas de física y química, así como a las ciencias básicas de la ingeniería. Los modelos matemáticos de tercera generación se localizan en las ciencias de aplicación de la ingeniería, aunque algunos también están en las ciencias básicas de la ingeniería. Mientras que los modelos matemáticos de cuarta generación están presentes en las asignaturas de aplicación de la ingeniería.

La correlación que se establece entre el tipo de asignatura de la ingeniería que cursará del estudiante y el tipo de generación del modelo matemático, permite determinar en qué semestre escolar se usarán los modelos matemáticos de una generación determinada.

Con este conocimiento se da pertinencia a los eventos contextualizados que son incorporados en la estrategia didáctica de la Matemática en Contexto. Así, se pueden ir dosificando los eventos contextualizados que se presentan a los alumnos y que son accesibles a ellos según el semestre escolar en que están inscritos.

En el siguiente cuadro se resumen la caracterización y clasificación de los modelos matemáticos en ingeniería.

\begin{tabular}{|c|c|c|}
\hline \multicolumn{2}{|c|}{ CARACTERIZACIÓN DE LOS MODELOS MATEMÁTICOS } \\
\hline $\begin{array}{c}\text { Modelaje de objetos } \\
\text { de la ingeniería }\end{array}$ & Modelaje de problemas de la ingeniería \\
\hline $\begin{array}{c}\text { La clasificación está en } \\
\text { función del uso que } \\
\text { le da la ingeniería }\end{array}$ & $\begin{array}{c}\text { La clasificación está en función de } \\
\text { las áreas cognitivas de la ingeniería }\end{array}$ \\
\hline
\end{tabular}

R. B. E. C. T., vol 5, núm. 3, set-dez.2012 ISSN - 1982-873X 


\begin{tabular}{|c|c|c|c|c|c|}
\hline $\begin{array}{l}\text { Modelos } \\
\text { estáticos }\end{array}$ & $\begin{array}{c}\text { Modelos } \\
\text { dinámicos }\end{array}$ & $\begin{array}{c}\text { Modelos } \\
\text { de primera } \\
\text { generación }\end{array}$ & $\begin{array}{c}\text { Modelos } \\
\text { de segunda } \\
\text { generación }\end{array}$ & $\begin{array}{c}\text { Modelos } \\
\text { de tercera } \\
\text { generación }\end{array}$ & $\begin{array}{c}\text { Modelos } \\
\text { de cuarta } \\
\text { generación }\end{array}$ \\
\hline
\end{tabular}

CUADRO. Clasificación de los modelos matemáticos en ingeniería

\section{Experiencias con la matemática en contexto}

Se comentarán dos experiencias que muestran las ventajas de la estrategia didáctica de la matemática en contexto, la cual incluye la modelación matemática.

En la primera experiencia se montó un experimento en donde fue impartido un curso sobre el análisis de Fourier en el contexto del análisis de señales eléctricas y electromagnéticas al grupo A, mientras que de forma simultánea el mismo tema se impartió en el grupo B de forma tradicional (Camarena, 1993; 2005).

Las evaluaciones, bajo un mismo instrumento de evaluación, de ambos grupos al finalizar los cursos no fueron tan diferentes, se consideró que la diferencia no era significativa, aunque cabe mencionar que el grupo A (el que recibió la matemática en contexto) obtuvo mejores calificaciones que el grupo $B$, quien recibió un curso tradicional.

Se les hizo un seguimiento a ambos grupos y después de dos semestres, cuando cursaban la asignatura de Comunicaciones I: análisis de señales (la disciplina del contexto), los resultados eron asombrosos, los alumnos del grupo A podían modelar matemáticamente con destreza las señales que se les proporcionaban, tanto en el dominio del tiempo como en el de la frecuencia, e interpretaban las características de las señales a partir de las expresiones matemáticas sin dificultad (se podría decir que con familiaridad).

Mientras que el grupo B tuvo un comportamiento igual que todos los demás estudiantes que están en el mismo semestre de la carrera y dos semestres atrás cursaron el tema de análisis de Fourier. Es decir, para ellos la herramienta matemática parece que nunca la han recibido, las señales no podían modelarlas matemáticamente, las características de las señales no podían predecirlas, tal parecía que no tenían las bases que se suponía les había brindado su curso de matemáticas sobre el análisis de Fourier y los conocimientos sobre señales que proporcionaban sus cursos básicos de la ingeniería.

La segunda experiencia relata el caso de un grupo piloto de la carrera de Ingeniería en Comunicaciones y Electrónica de la Escuela Superior de Ingeniería Mecánica y Eléctrica campus Zacatenco del Instituto Politécnico Nacional en México, en el cual se trabajó todo el proceso metodológico que describe la fase didáctica de la Matemática en el Contexto de las Ciencias (para conocer todo el proceso metodológico se pueden ver las referencias de Camarena (2000; 
2007; 2008). El trabajo con el grupo piloto permitió ver el éxito de la estrategia didáctica en su formación integral.

El $70 \%$ de estos estudiantes fueron a realizar estudios de posgrado a otros países, de los cuales no los dejaron regresar, los invitaron a trabajar con los investigadores de los países de estudio.

Las industrias en las que trabajaron los demás estudiantes, fueron empresas que hicieron llegar un reconocimiento a la Escuela por la calidad de los ingenieros egresados, los cuales resolvían satisfactoriamente los problemas de la empresa realizando modelación matemática.

\section{Conclusiones}

Como ha sido mostrado, la modelación matemática forma parte de la estrategia didáctica de la Matemática en Contexto. Con ésta se le va preparando al estudiante para que enfrente su tura actividad laboral y profesional de forma competente.

El contar con una clasificación de los modelos matemáticos como la que se ha presentado, en donde éstos se correlacionan con las asignaturas del estudiante, se permite dar pertinencia a la modelación matemática que gradualmente aprenderá el estudiante.

Si la modelación matemática se incorporara desde los niveles básicos de estudio la problemática tendría otra dimensión; ya que, como comenta Mochón (1997), la modelación matemática se puede llevar a todos los niveles educativos.

\section{BIBLIOGRAFÍA}

Ausubel David P., Novak Joseph D. y Hanesian Helen, Psicología educativa, un punto de vista cognoscitivo. Editorial Trillas, 1990.

Camarena G. Patricia, El currículo de las matemáticas en ingeniería. Memorias de las Mesas redondas sobre definición de líneas de investigación en el IPN, México, 1984.

- Diseño de un curso de ecuaciones diferenciales en el contexto de los circuitos eléctricos. Tesis de Maestría en Ciencias con especialidad en Matemática Educativa, CINVESTAV-IPN, México, 1987.

- Especialidad en docencia de la ingeniería matemática en electrónica. Editorial ESIME-IPN, México, 1990.

- Curso de análisis de Fourier en el contexto del análisis de señales eléctricas. Editorial ESIME-IPN, México, 1993.

R. B. E. C. T., vol 5, núm. 3, set-dez.2012 ISSN - 1982-873X 
- La enseñanza de las matemáticas en el contexto de la ingeniería. Memorias del XXVIII Congreso Nacional de la Sociedad Matemática Mexicana, México, 1995.

- Hacia la integración del conocimiento: Matemáticas e ingeniería. Memorias del 2oㅡ Congreso Internacional de Ingeniería Electromecánica y de Sistemas, México, 1999.

- Reporte del proyecto de investigación titulado: Etapas de la matemática en el contexto de la ingeniería. ESIME-IPN, México, 2000.

- Reporte del proyecto de investigación titulado: Los modelos matemáticos como etapa de la matemática en el contexto de la ingeniería. ESIME-IPN, México, 2001.

- Reporte del proyecto de investigación titulado: La matemática en el contexto de las ciencias: las competencias profesionales. ESIME-IPN, México, 2005.

- Reporte del proyecto de investigación titulado: La matemática formal en la modelación matemática. ESIME-IPN, México, 2007.

- La matemática en el contexto de las ciencias. Memorias del III Coloquio Internacional sobre Enseñanza de las Matemáticas, Lima, Perú, 2008.

Polya G. Cómo plantear y resolver problemas. Editorial Trillas, 1976.

Santos Luz Manuel Principios y métodos de la resolución de problemas en el aprendizaje de las matemáticas. Grupo Editorial Iberoamérica, Serie Didáctica, México, 2000.

Mochón Simón Modelos matemáticos para todos los niveles. Actas de la undécima Reunión Latinoamericana de Matemática Educativa, Grupo Editorial Iberoamérica, México, 1997.

Patricia Camarena Gallardo - ESCUELA SUPERIOR DE INGENIERIA MECANICA Y ELECTRICA (UNIDAD ZACATENCO) - pcamarena@ipn.mx 